\title{
Unwanted pregnancy and associated factors among female students of Madawalabu University Bale Zone, Oromia Region south east, Ethiopia
}

\author{
Motuma Getachew Erena ${ }^{1,}$, Amene Abebe Kerbo² \\ ${ }^{1}$ Department of Nursing, College of Medicine and Health Science, Wellaga University, Nekemite, Ethiopia \\ ${ }^{2}$ Department of Nursing, College of Medicine and health Science, Madawalabu University, Bale Goba, Ethiopia \\ Email address: \\ Motyamo@Gmail.Com (M. G. Erena), ameneabe@gmail.com (A. A. Kerbo)

\section{To cite this article:} \\ Motuma Getachew Erena, Amene Abebe Kerbo. Unwanted Pregnancy and Associated Factors among Female Students of Madawalabu \\ University Bale Zone, Oromia Region South East, Ethiopia. Science Journal of Public Health. Vol. 3, No. 1, 2015, pp.50-55. \\ doi: $10.11648 /$ j.sjph.20150301.19
}

\begin{abstract}
Globally as well as in the developing world unintended pregnancies are among the major social and health problems. In Ethiopia, 54\% of pregnancies to girls under age 15 are unwanted. In some countries where accesses to safe and legal abortion services are restricted, unsafe abortion is the cause for more than $30 \%$ of maternal deaths. The aim of this study was to assess factors associated with unwanted pregnancy among female students of Madawalabu University from 01-21, March 2014. A cross sectional study was conducted among 814 female students of Madawalabu university drawn randomly from 6 selected schools in 01-21, March 2014. Self administered questionnaire, adapted from previous relevant studies was used to assess Factors associated with unwanted pregnancy among female students of Madawalabu University. Data was entered, cleaned and then analyzed using Bivariate and multivariate logistic regression models. A total of 814 female students responded to the questionnaire adequately making the response rate of $96 \%$. The mean age of study participants was 20.74 years with standard deviation of 1.47 . The overall prevalence of unwanted pregnancy among those who ever had sexual intercourse was $8.1 \%$ and among all study participants it was $32.4 \%$. Ever hearing about family planning, consistent use of family planning in the last twelve months, and drinking alcohol in the last one week were among significantly associated with unwanted pregnancy Thus, Madawalabu University HIV/AIDS prevention and control office and other stakeholders are recommended to promote extensively family planning utilization to avoid unwanted pregnancies.
\end{abstract}

Keywords: Unwanted Pregnancy, Students, Family Planning

\section{Introduction}

Today's generation of adolescents is the largest in history. Nearly half of the global population is less than 25 years old (UNFPA, 2006). These young people face many significant sexual reproductive health challenges such as limited access to youth friendly services including information on growth, sexuality and family planning. This has led youth into risky sexual behaviors resulting to high STI and HIV prevalence, early pregnancy and vulnerability to delivery complications resulting in high rates of death and disability $[1,2]$.

Negative outcomes of early pregnancy and sexually transmitted infections (STIs), including HIV/AIDS, threaten the health of people in the second decade of life more than any other age group. At the same time, adolescents are the greatest hope for turning the tide against STIs, AIDS, and early pregnancy [3].

Biologically, the immature reproductive and immune systems of adolescent girls translate to increased susceptibility to STIs and HIV transmission; pregnancy and delivery for those with incomplete body growth exposes them to problems that are less common in adult women [4].

Girls who get pregnant at the age of 14 years or younger, and, to a lesser extent, at 15-17 years and 18-19 years, are at considerably heightened risk of complications such as pregnancy-induced hypertension, obstructed and prolonged labour, vaginal tearing, obstetric fistula, and postpartum hemorrhage, in addition to premature delivery, low infant birth weight, perinatal mortality and health problems in the newborn [5]. 
Both married and unmarried adolescents experience unplanned and unwanted pregnancies and births, even in countries where early marriage and childbearing are the norm [6].

Unwanted pregnancy is one of the major RH challenges faced by adolescents in Ethiopia. As, 54\% of pregnancies to girls under age 15 are unwanted (wanted later or not wanted) compared to $37 \%$ for those ages $20-24$ [7]

Unwanted pregnancies in youths and complications of induced abortions are an important health problem in the world. Each year, approximately 20 million abortions are performed worldwide, $95 \%$ of them in developing countries [8].The most significant current discussions about unsafe abortion and unwanted pregnancy are the denial of women's health rights and the disproportional number of maternal deaths in developing countries [9].

Unintended pregnancy, which includes both mistimed and unwanted pregnancies, is a global social and health challenge. Worldwide, $38 \%$ of pregnancies are unintended (that is, some 80 million pregnancies annually). In sub- Saharan Africa, unintended pregnancy accounts for more than a quarter of the 40 million pregnancies that occur annually $[5,10]$.

Young women have higher likelihood of inconsistent or nonuse of effective family planning methods than older women and have greater risk to have mistimed than intended pregnancy $[11,12]$.

Studies conducted in various developed and developing Countries revealed that unintended pregnancies can have serious health, social, and economic consequences. The negative consequences of unwanted pregnancies are increased risk of low birth weight and of being born prematurely; as a result, infants have a high risk of mortality $[13,14,15]$.

\section{Method and Materials}

\subsection{Study Setting and Participation}

Institutional based cross-sectional study was carried out. Goba and robe towns are found in south-eastern part of Ethiopia. Goba and robe towns are found, Bale Zone of the Oromia Region approximately $432 \& 445 \mathrm{~km}$ far from Addis Ababa respectively. Madawalabu University is one of the governmentally established universities in 1999 E.C in bale zone. Currently the university is training students in 9 schools, one institute and one college in the basis of regular and continuing education programs. This study was conducted in 01-21, march 2014. All female students of Madawalabu University enrolled in the regular program were the source population for this study and female students of selected schools and college of medicine and health sciences of Madawalabu University were the study population

\subsection{Sample Size Determination and Sampling Methods}

The required sample size was determined by using single population proportion formula with the following assumptions:
- A $95 \%$ confidence interval

- $50 \%$ population proportion

- $5 \%$ marginal error

- Design effect of 2

$$
\mathrm{n}=\frac{\mathrm{Z}^{2} \mathrm{P}(1-\mathrm{P}) \times \mathrm{D}}{\mathrm{d}^{2}}=\frac{1.96^{2} 0.5(0.5) \times 2}{0.05^{2}}=770
$$

Where, $\mathrm{n}=$ sample size, $\mathrm{p}=$ proportion, $\mathrm{d}=$ margin of error, $\mathrm{D}=$ design effect.

Including $10 \%$ non response rate the total sample size become $n=847$.

First Stratified random sampling technique was applied by assuming there is knowledge difference about unwanted pregnancy and abortion law of Ethiopia between health and non health school students of Madawalabu University. Then the total sample size was proportionally allocated to health and non health schools. Schools were selected by simple random sampling technique using lottery method. Students list was obtained from Madawalabu University main registrar Then we gave an orderly code for the specific student. Finally, we used the computer generated sample of study participant female students to fill the questionnaire prepared for the study.

\subsection{Data Collection Procedure}

Data were collected by self-administered questionnaire method using structured and pretested questionnaire. Five data facilitators and two supervisors were recruited and training was given for them for 2 days before the actual data collection on the objectives, relevance of the study, and confidentiality of the information, respondent's right and informed consent. Moreover, practical exercise was done by data collectors during training with the principal investigators how to introduce the above mentioned issues. Data collection was done by arriving at the end of the scheduled time based on the block number, lecture room number and schedule that was obtained from school director's office. Supervision of the data collection process was done by supervisors along with the principal investigators.

\subsection{Data Quality Control}

The questionnaire was prepared in English then translated to Amharic and Afan Oromo languages and translated back to English language Two supervisors with first degree in Health sciences with the principal investigators supervised the data collection process. Training was given to data collectors and the supervisors before the actual data collection. The questionnaire was pre-tested two weeks before the start of the actual data collection at school of social sciences. After pretest, questions were revised, edited, and those found to be unclear were modified.

\subsection{Data Management and Analysis}

The collected Data by using structured and pretested questionnaire was coded, entered and cleaned for its completeness and errors, and then analysis was done using 
SPSS version 16 statistical soft ware packages. Relevant variables were explained by frequency tables, graphs and summary statistics. Association between dependent and independent variables was assessed by both Binary and multiple logistic regressions and its strength of association was presented using odds ratio and $95 \%$ confidence intervals.

\subsection{Ethical Consideration}

Ethical clearance and approval was obtained from research and community service directorate Madawalabu University. Permission was obtained from academic vice president and registrar and alumni directorate. Oral informed consent obtained from participants. Confidentiality and anonymity ensured. Participants were informed that their participation was voluntary.

\section{Result}

\subsection{Socio-Demographic Characteristics of Study Participants}

Table 1. Socio Demographic Characteristics of female students, Madawalabu University, Bale, Ethiopia, March, 2014

\begin{tabular}{|c|c|c|}
\hline Characteristics & Number & Percent \\
\hline \multicolumn{3}{|l|}{ Age } \\
\hline $15-19$ & 121 & 14.9 \\
\hline $20-24$ & 661 & 81.2 \\
\hline $25-29$ & 32 & 3.9 \\
\hline \multicolumn{3}{|l|}{ Religion } \\
\hline Orthodox & 478 & 58.7 \\
\hline Protestant & 193 & 23.7 \\
\hline Muslim & 125 & 15.4 \\
\hline Catholic & 13 & 1.6 \\
\hline Other* & 5 & 0.6 \\
\hline \multicolumn{3}{|l|}{ Schools } \\
\hline Engineering and technology & 438 & 53.8 \\
\hline Business and economics & 146 & 17.9 \\
\hline Health and medicine & 115 & 14.1 \\
\hline Language studies & 57 & 7 \\
\hline Behavioral sciences & 40 & 4.9 \\
\hline Agriculture & 18 & 2.2 \\
\hline \multicolumn{3}{|l|}{ Ethnicity } \\
\hline Oromo & 495 & 60.8 \\
\hline Amhara & 213 & 26.6 \\
\hline Tigre & 49 & 6 \\
\hline Wolyta & 25 & 3.1 \\
\hline Guraghe & 15 & 1.8 \\
\hline Other\# & 17 & 2.1 \\
\hline \multicolumn{3}{|l|}{ Marital status } \\
\hline Single & 751 & 92.3 \\
\hline Married & 172 & 7.7 \\
\hline \multicolumn{3}{|l|}{ Monthly pocket money } \\
\hline$<330 \mathrm{~EB}$ & 582 & 65.4 \\
\hline$>=330 \mathrm{~EB}$ & 282 & 34.6 \\
\hline
\end{tabular}

*Adventist and wakefeta are other religions that werereported

\# Sidama,kembata,hadiya were other ethnicity reported
From the total 847 female students of MWU recruited, 814 responded to the questionnaire adequately making the response rate of $96 \%$. The mean age of study participants was $20.74 \pm 1.47 \mathrm{SD}$ years. From the total female students 665 $(87.4 \%)$ were in the age group of 20-24 years and 32 (3.9\%) of the participants were in the age range of 25-29 years.

Oromo and Amhara were the major ethnic groups which account $495(60.8 \%)$ and $213(26.2 \%)$, respectively. Four hundred seventy eight $(58.7 \%)$ of the participants were Orthodox religion followers followed by Protestant Christianity 193(23.7\%). Seven hundred forty nine (92\%) of the study participants reported to be single while $63(7.7 \%)$ were married. Four hundred thirty eight $(53.8 \%)$ and $146(17.9 \%)$ female students were from engineering and technology schools followed by business and economics school respectively and the rest were from college of health and medicine, school of language studies, school of behavioral science and school agriculture. The mean monthly pocket money the students reported they were getting was $330.22 \mathrm{~EB}$.five hundred thirty two (65.4\%) of the participants reported they get above average monthly pocket money and the rest get below the average (Table-1)

\subsection{Family Planning}

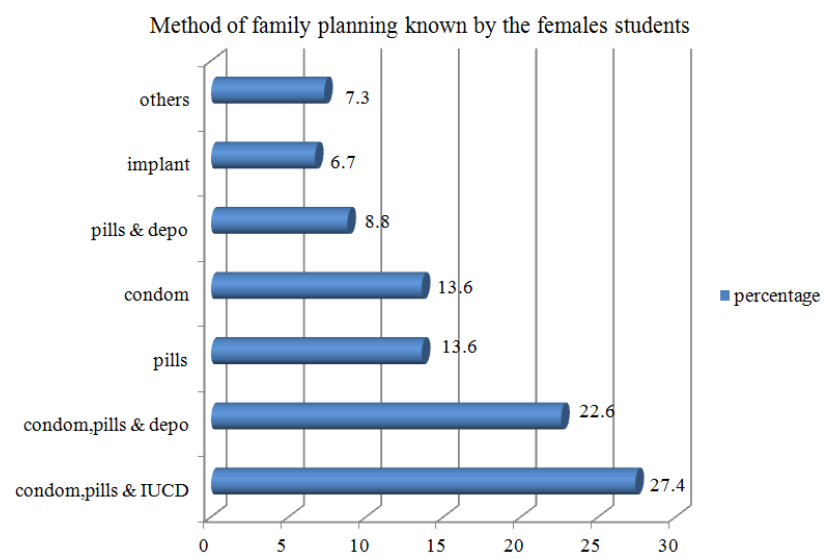

Figure 1. Method of family planning known by female Madawalabu university students, Bale, Ethiopia, March, 2014.

According to this study $610(74.9 \%)$ of the female students ever had heard about family planning. The most commonly mentioned family planning method were condom, pills and IUCD $138(27.4 \%)$ followed by condom, pills and depoprovera $167(22.6 \%)$.

\subsection{Past Sexual History}

Regarding the past sexual history only $136(16.7 \%)$ of the females ever had sexual intercourse. The median age for sexual debut was $18 \pm 3.6$ SD years. Among those who ever had sexual intercourse $38(27.9 \%)$ started sexual intercourse after joining the campus. The abundant reason reported for initiation of first sexual intercourse were personal desire $83(61 \%)$ followed by peer influence $40(29.4 \%$ ) (Table 2$)$. 
Table 2. Past sexual history of female Madawalabu University, Bale, Ethiopia, and March, 2014

\begin{tabular}{lll}
\hline characteristics & Frequency & Percentage \\
\hline Ever had sexual intercourse & 136 & \\
yes & 678 & 83.3 \\
no & 814 & 100 \\
Total & & \\
Reason for having sexual intercourse & & 61 \\
personal desire & 83 & 29.4 \\
peer pressure & 40 & 0.8 \\
Influence of substance use & 1 & 4.4 \\
forced & 6 & 4.4 \\
other & 6 & 100 \\
Total & 136 & \\
Time of initiation of sex & & 72.1 \\
before joining university & 98 & 27.9 \\
after joining university & 38 & 100 \\
Total & 136 & \\
\hline
\end{tabular}

Table 3. Current sexual history of female Madawalabu University students, Bale, Ethiopia, March, 2014

\begin{tabular}{lll}
\hline characteristics & frequency & percentage \\
\hline Last twelve month sexual intercourse & & \\
yes & 107 & 78.7 \\
no & 29 & 21.3 \\
Total & 136 & 100 \\
Frequency of sexual intercourse & & \\
once & 35 & 32.7 \\
some times & 63 & 58.9 \\
usually & 9 & 8.4 \\
Total & 107 & 100 \\
Pattern of contraceptive usage & & \\
consistently used & 68 & 63.5 \\
not used & 39 & 36.5 \\
Total & 107 & 100 \\
Method of family planning used & & \\
pills & 34 & 46.6 \\
condom & 9 & 12.4 \\
depo & 25 & 34.2 \\
implants & 3 & 4.1 \\
IUCD & 2 & 2.7 \\
Total & 73 & 100 \\
reason for inconsistent use of contraceptive & \\
sex partner opposition & 6 & 15.4 \\
fear to go to clinic to buy & 30 & 76.9 \\
other & 3 & 7.7 \\
Total & 39 & 100 \\
\hline
\end{tabular}

This study revealed from those who ever had sexual intercourse $107(78.7 \%$ ) of them were sexually active in the last twelve months. Sixty eight $(63.6 \%)$ were used family planning method consistently while the rest were not. Among the consistent users the most common type of contraceptive used were pills(45.1\%) and depo-provera (35.1\%).The paramount justification stated by more than third quartiles(76.9.\%) for not complying with family planning methods was fear to go to clinic to buy contraceptives followed by partner opposition(15.4\%) (Table 3)

\subsection{History of Pregnancy}

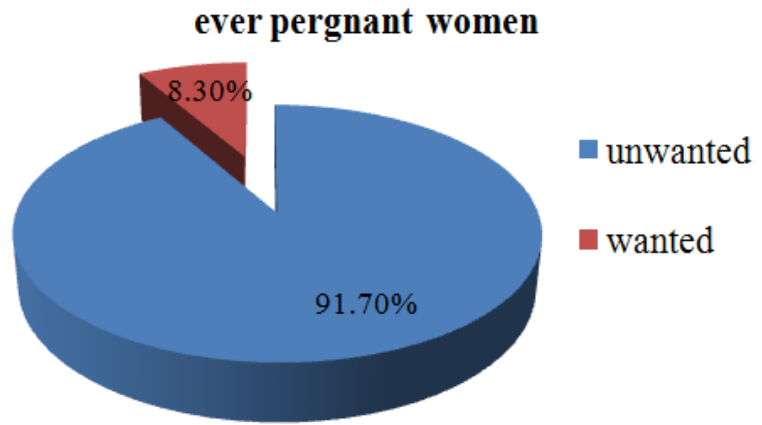

Figure 2. Prevalence of pregnancy among female Madawalabu university students, Bale, Ethiopia, March, 2014.

From all respondents $12(1.5 \%)$ had ever been pregnant and almost more than $90 \%$ of this pregnancies were reported as they were unwanted. The median age at first pregnancy was $19 \pm 1.6 S D$ years. The overall prevalence of unwanted pregnancy among those who ever had sexual experience was $11(8.1 \%)$ while $11(1.4 \%)$ from total respondents. The main reason mentioned for unwanted pregnancy was the need to continue their education $(80 \%)$.

\subsection{Factors Associated with Unwanted Pregnancy among Female Madawalabu University Students}

On the bivariate analysis, ever hearing about family planning, frequency of sexual intercourse in the last 12 months, consistent use of family planning in the last twelve months, unexpected sexual intercourse, ever hearing about emergency contraceptives and drinking alcohol in the last one week identified to be significantly associated with unwanted pregnancy. But, ever hearing about family planning, consistent use of family planning in the last twelve months, unexpected sexual intercourse, ever hearing about emergency contraceptives and drinking alcohol in the last one week were remained significantly associated with unwanted pregnancy in the multiple logistic regression analysis. Unwanted pregnancy was lower among those who ever heard about family planning, consistently used family planning in the last twelve month and ever heard about emergency contraceptive. those who ever heard about family planning were $97.4 \%$ less likely to have unwanted pregnancy as compared to those who didn't heard about family planning $[\operatorname{AOR}(95 \% \mathrm{CI})=0.026(0.004,0.196)]$. Those who were consistently used family planning in the last twelve month were also $96 \%$ less likely to have unwanted pregnancy as compared to those who failed to use consistently [AOR $(95 \% \mathrm{CI})=0.04(0.003,0.558)]$. Those who ever heard about emergency contraceptive were $93 \%$ less likely exposed for unwanted pregnancy as compared to those who didn't heard about emergency contraceptives $\quad[\mathrm{AOR}(95 \% \mathrm{CI})=$ 0.07(0.001,19.71)]

Unwanted pregnancy was 7.82 times higher among study participants who encountered unexpected sexual intercourse when compared to those who didn't encounter [AOR $(95 \% \mathrm{CI})=7.82(1.72,23.51)]$. In addition, those female 
students who drank alcohol in the last one week previous to this study were 8.43 times more likely to have unwanted

pregnancy as compared to those who didn't drink alcohol. [AOR $(95 \% \mathrm{CI})=8.43(1.82,29.77)]$ (Table 4$)$.

Table 4. Factors associated with unwanted pregnancy among female Madawalabu University students, Bale, Ethiopia, and March, 2014.

\begin{tabular}{|c|c|c|c|c|}
\hline \multirow{2}{*}{ Characteristics } & \multicolumn{2}{|c|}{ unwanted pregnancy } & \multirow{2}{*}{ COR (95\%) } & \multirow{2}{*}{ AOR (95\%) } \\
\hline & yes & No & & \\
\hline \multicolumn{5}{|c|}{ Heardabout family planning } \\
\hline yes & 5 & 109 & $0.122(0.033,0.448)$ & $0.026(0.004,0.196)^{*}$ \\
\hline no & 6 & 16 & 1 & 1 \\
\hline \multicolumn{5}{|c|}{ Frequencyof sexual intercourse in thelast 12 months } \\
\hline once & 1 & 35 & 1 & 1 \\
\hline sometimes & 1 & 65 & $1.806(0.110,29.738)$ & $1.49((0.26,21.47)$ \\
\hline usually & 5 & 9 & $0.050(0.005,0.483)$ & $0.02(0.003,14.34)$ \\
\hline \multicolumn{5}{|c|}{ Consistentuse of family planning in thelast 12 months } \\
\hline Yes & 1 & 66 & $0.083(0.010,0.72)$ & $0.04(0.003,0.558)^{*}$ \\
\hline No & 6 & 33 & 1 & 1 \\
\hline \multicolumn{5}{|c|}{ Unexpectedsexual intercourse } \\
\hline yes & 6 & 19 & $6.69(1.85,24.16)$ & $7.82(1.72,23.51)^{*}$ \\
\hline no & 5 & 106 & 1 & 1 \\
\hline \multicolumn{5}{|c|}{ Everheard aboutemergency contraceptive } \\
\hline Yes & 2 & 69 & $0.18(0.037,0.89)$ & $0.07(0.001,19.71)^{*}$ \\
\hline no & 9 & 56 & 1 & 1 \\
\hline \multicolumn{5}{|c|}{ Drinkingalcohol in the last week } \\
\hline yes & 6 & 18 & $7.13(1.97,25.85)$ & $8.43(1.82,29.77)^{*}$ \\
\hline no & 5 & 107 & 1 & 1 \\
\hline
\end{tabular}

*Statistically significant $(\mathrm{P} \leq 0.05)$

\section{Discussion}

Due to various reasons, youth are vulnerable to unplanned and unintended early sexual encounters which lead to unwanted pregnancies. Therefore, they are exposed to seek for induced abortion and candidates to suffer its complications. The youth who undergo an induced abortion expose themselves to serious health risks such as hemorrhage, genital injuries, sepsis and death $[16,17]$.

In this study from total respondents about $12.6 \%$ female students were sexually active in the last twelve months. But, study done in woliatta Soddo University among female students indicates that close to a quarter of respondents $(23.7 \%)$ were sexually active. This difference might be attributed to the time laps between the studies [18].

Unplanned pregnancies may be prevented by using contraceptive methods, such as the oral contraceptive pills, the long-term hormonal injections, condoms, tubal ligation or a vasectomy (ContraceptiveChart2008).In this study, the contraceptive utilization result is in line with national survey conducted in Finland universities where majority of the females was using pills to avoid pregnancy. On contrary to this, in the study conducted in Ugandan university more than half of the females were using condoms as contraception. This might be due to the increased awareness of females in this university on dual protection of condom. On the other hand, the main reasons dictated in this study for not using family planning method were nearly same as the study conducted in Ugandian universities. [19].

This study revealed that those who ever had been pregnant were less than $2 \%$ while in study conducted in woliata Soddo
University it was $7.7 \%$. This might be due to sample size difference or the time lapse between the studies. But, in both studies more than $85 \%$ of the pregnancies were unwanted [18].

Unwanted pregnancy was 7.82 times higher among study participants who encountered unexpected sexual intercourse when compared to those who didn't encounter. This result might be due to lack of awareness of the later group on emergency contraceptive or low perception of risk of pregnancy.

Those who ever heard about emergency contraceptive were $93 \%$ less likely exposed for unwanted pregnancy as compared to those who didn't heard about emergency contraceptives. This might be due to those who heard about the emergency contraceptive they use it when they encounter unexpected sexual intercourse for prevention of unwanted pregnancy.

Those female students who drink alcohol last week before the study were 8.4 times more likely to have had unwanted pregnancy than those who never drank alcohol last week. This might be due to the consumption of alcohol that may blur their decision making on utilization of contraceptive to avoid pregnancy.

\section{Conclusion}

Nearly three quarter of study participants were ever heard about family planning. The numbers of sexually active female students were low but it is still of public health concern. Even though, most of those sexually active females use contraceptive consistently, considerable portion of them 
weren't using it consistently. Among those who used the contraceptive consistently most of them uses pills and inject able as contraceptives. Almost one fifth of the sexually active study subjects encounter unexpected sexual intercourse with more than two third of them reported that they took post pills to avoid pregnancy. Less than two percent of sexually active females were ever pregnant from which more than ninthly percent of them being reported as unwanted. The main reason mentioned for the unwanted pregnancy was to continue their education.

\section{Strength and Weakness}

The study addressed previously unscathed area which is of great public health concern. Authors of this research feel confident the study can provide base line evidence for addressing sexual and reproductive health problems within Madawalabu University and other similar universities in Ethiopia. Even though the objectives of the study explained clearly still there may be possibility of Social desirability bias. There were also some sensitive issues that couldn't be addressed by quantitative research deign.

\section{Authors' Contributions}

MG \& AA conceived and designed the study. MG \& AA analyzed the data and interpreted the results.

\section{Acknowledgements}

We would like to thank Madawalabu University that, this research would not have been possible without the financial support of it. Our thanks also extend to all staffs of Madawalabu university who participated by commenting the research work. Finally, the researchers would like to acknowledge study participants for their patience in responding the questions scarifying their time.

\section{References}

[1] African Youth Alliance/ Pathfinder International. Scaling up youth friendly services, Concept paper. African Youth Alliance/ Pathfinder International, 2002.

[2] African Youth Alliance/ Pathfinder International. Youth friendly sexual and reproductive health services: An assessment of facilities. African Youth Alliance/ Pathfinder International, 2003.

[3] UNAIDS,A report of the global AIDS epidemic. http://www.unaids.org,2006.
[4] Dehne KL, RiednerG, Sexually transmitted infections among adolescents: the need for adequate health services. Geneva: World Health Organization, 2005.

[5] World Health Organization. Issues in adolescent health and development: Adolescent pregnancy.Geneva, 2003 (unpublished).

[6] Singh S. Adolescent childbearing in developing countries: a global review. Studies in Family Planning, Vol. 29, 117-13, 1998.

[7] FMOH, National reproductive health strategy, 2006-2015.

[8] World Health Organization, Unsafe abortion: global and regionalestimatesoftheincidenceofunsafeabortionandassociate dmortality in $2008,6^{\text {th }}$ Edition. Department of Reproductive Health and Research, World Health Organization, 2011.

[9] Grimes David A, Benson J, Singh S, Romero M, Ganatra B, Okonofua Friday E et al. Unsafe abortion: the preventable pandemic. The Lancet Sexual and Reproductive Health Series 4, October 2006.

[10] Eggleston E.Determinants of unintended pregnancy among women in Ecuador. IntFam Plan Perspect, 25(1):27-33, 1999.

[11] Kost K, Forrest JD Intentions status of US births in 1988: differences by mother's socio demographic characteristics. FamPlannPerspect, vol.27 (1):11-17, 1995.

[12] Williams L(1991).Determinants of unwanted childbearing among ever married women in the United States. FamPlannPerspect, vol.23(5): 212-221,1973-1988

[13] Asling-Monemi K, Pena R, Ellsberg M, Persson L. Violence against women increases the risk of infant and child mortality: a case-referent study in Nicaragua. Bulletin of the World Health Organization, 81:10-18, 2003.

[14] Bacci A et al,Outcome of teenage pregnancy in Maputo, Mozambique. International Journal of Gynecology and Obstetrics, 40:19-23, 1993.

[15] Balde $M$ et al, Improvement of the services in reproductive health for adolescents in Guinea. $7^{\text {th }}$ Congress of the African Society of Gynaecology and Obstetrics in Bamako, Mali.2003

[16] MbonyeAK,Abortion in Uganda:magnitude and implications.Afr. J. Reproductive. Health 4(2):104, 2000.

[17] Silberschmidt M, Rasch.Adolescent girls, illegal abortion and "Angar-laddies" in Dar-es-Salam: Vulnerable victims and active social agents. Soc. Sci. Med. 52(12):1815-26. 2001.

[18] Amha A.G,Kalemelekot N.T, Tesfa.M. Magnitude and risk factors of abortion among regular female students in WolaitaSodo University, Ethiopia. BMC Women's Health14:50, 2014.

[19] DevikaMehra, Per-OlofÖstergren, Björn Ekman, AnetteAgardh Inconsistent condom use among Ugandan university students from a gender perspective: a crosssectional study.7, 2014. 